\title{
Evaluation of the tone and viscoelastic properties of the masseter muscle in the supine position, and its relation to age and gender
}

\author{
Engin Ramazanoglu ${ }^{1, A-D, F}$, Begumhan Turhan ${ }^{2, A, C-F}$, Serkan Usgu ${ }^{2, A, C-F}$ \\ ${ }^{1}$ Department of Physiotherapy and Rehabilitation, Faculty of Health Sciences, İönü University, Malatya, Turkey \\ ${ }^{2}$ Department of Physiotherapy and Rehabilitation, Faculty of Health Sciences, Hasan Kalyoncu University, Gaziantep, Turkey \\ A - research concept and design; $\mathrm{B}$ - collection and/or assembly of data; $\mathrm{C}$ - data analysis and interpretation; \\ $D$ - writing the article; $E$ - critical revision of the article; $F$ - final approval of the article
}

Address for correspondence

Begumhan Turhan

E-mail: begum.aliosmanoglu@hku.edu.tr

Funding sources

None declared

Conflict of interest

None declared

Received on November 29, 2020

Reviewed on January 6,2021

Accepted on January 7, 2021

Published online on June 2, 2021

Cite as

Ramazanoglu E, Turhan B, Usgu S. Evaluation of the tone and viscoelastic properties of the masseter muscle in the supine position, and its relation to age and gender. Dent Med Probl. 2021;58(2):155-161. doi:10.17219/dmp/132241

DOI

$10.17219 / \mathrm{dmp} / 132241$

Copyright

○) 2021 by Wroclaw Medical University

This is an article distributed under the terms of the

Creative Commons Attribution 3.0 Unported License (CC BY 3.0)

(https://creativecommons.org/licenses/by/3.0/).

\begin{abstract}
Background. The masseter muscle can be evaluated in various ways to examine its condition in healthy individuals or to identify pathological changes in the muscle.

Objectives. This study aimed to examine the tone and viscoelastic parameters of the masseter muscle, which is the focal muscle of various pathologies, to reveal its relationship with age and gender, and to determine the reference values of this muscle in healthy individuals.

Material and methods. Individuals aged 18-50 years were evaluated. They were divided into 3 groups in terms of age. A total of 389 individuals participated in the study (18-28 years: 131 males, 104 females; 29-39 years: 29 males, 56 females; and $40-50$ years: 30 males, 39 females). The tone and viscoelastic properties of the masseter muscle were evaluated bilaterally in the supine position.

Results. The mean age of all individuals was $28.64 \pm 9.68$ years. The masseter muscle tone was found to be higher in men than in women. The elasticity of the muscle was higher in women $(p<0.05)$. It was determined that the masseter muscle tone and stiffness increased, whilst its elasticity decreased with aging $(p<0.05)$. A weak positive correlation was found between the right and left masseter muscle tone and age $(r=0.307$ and $r=0.325$, respectively; $p=0.001)$. There was a moderate positive correlation between the right and left masseter muscle stiffness and age $(r=0.507$ and $r=0.511$, respectively; $p=0.001)$. A strong positive correlation was observed between the right and left masseter muscle elasticity and age ( $r=0.614$ and $r=0.645$, respectively; $p=0.001$ ).
\end{abstract}

Conclusions. The data obtained in this study may assist clinicians in evaluating the treatment of the pathological conditions related to the masseter muscle as well as in the planning of treatment and pre- and post-operation evaluations.

Keywords: viscosity, aging, gender identity, muscle tone, masseter muscle 


\section{Introduction}

The masseter is one of the muscles of mastication, primarily responsible for the elevation and some protraction of the mandible. The masseter muscle thickness is extensively studied, as it is related to the craniofacial mechanisms. The activity of this muscle is also associated with chewing, swallowing, temporomandibular joint disorders (TMD), craniomandibular dysfunctions, bruxism, and orofacial pain. ${ }^{1-3}$ In a study by Ariji and Ariji, the formation of intramuscular echogenic bands (structures that reflect sound waves in ultrasound) was observed and the bands in the masseter muscle were thickened in patients with TMD. ${ }^{4}$ The masseter muscle can be evaluated in various ways to examine the condition of the muscle in healthy individuals or to identify pathological changes in the muscle. In recent studies, the masseter muscle was evaluated by means of electromyography (EMG), 5,6 ultrasonography (USG) ${ }^{7}$ and palpation. ${ }^{8}$ The muscle activity can be determined by placing EMG electrodes over the skin or EMG needles into the muscle. This method is invasive and can be painful. ${ }^{7}$ Modalities such as elastography, shear-wave elastography (SWE) or free oscillation techniques are valid and reliable in the evaluation of the mechanical properties of muscles and tendons. However, these modalities may have limited availability in clinics because of high purchasing and maintenance costs, and the requirement of technical expertise. ${ }^{9}$ Thus, there is a need for easy-to-use, cost-effective, objective, reliable, and valid methods to evaluate the mechanical properties of the musculoskeletal system. ${ }^{10}$ Palpation is a prominent and easy method for the evaluation of cases when digital measurements are not available. ${ }^{11}$ Masseter muscle palpation is a subjective and qualitative evaluation. On the other hand, the objective measurement of the viscoelastic properties of the muscle with Myoton ${ }^{\circledR}$ PRO (Myoton, Tallinn, Estonia) has high test-retest reliability. ${ }^{12,13}$ It has been observed that the myotonometric measurements obtained with EMG and USG show a high level of correlation with the measurements obtained with the Myoton PRO device. ${ }^{14}$

It has been stated in studies that the masseter muscle thickness, the masticatory function and the craniofacial mechanisms are related. Aging affects facial morphology, the muscle thickness, occlusal morphology, and bite force. It has also been reported that muscle anatomy is related to the physiognomy and anthropometric variables of individuals. There are some suggestions that the muscle thickness is related to facial morphology. Some authors emphasized that the thickness of the head and neck muscles, muscle pain, and facial morphology could be associated with bite force and occlusal factors. In addition, there seems to be a relationship between the masseter muscle thickness and various features of dental arches, such as the alveolar process thickness and the maxillary dental arch width. ${ }^{3,4,7}$ The activity of the muscle is associated with the chewing behavior and swallowing, and impaired activity may be associated with TMD. The masseter is the largest jaw-raising muscle and also provides the greatest contribution to jaw closure, and its size is closely related to bite force. It is known that variations in the size of this muscle may be a critical factor regarding individual differences in oral functions. ${ }^{3,7}$ We believe that the data obtained in this study may assist clinicians in evaluating the treatment of the pathological conditions related to the masseter muscle as well as in the planning of treatment and pre- and post-operation evaluations. There are several studies on chewing muscles that have evaluated the muscles with the use of the Myoton PRO device, but studies that focus on the masseter muscle are rare. This study aimed to examine the tone and viscoelastic parameters of the masseter muscle, which is the focal muscle of various pathologies, to reveal its relationship with age and gender, and to determine the reference values of this muscle in healthy individuals.

\section{Material and methods}

\section{Participants and the study design}

In our study, 420 individuals aged $18-50$ years were randomly selected from the students and staff of the Hasan Kalyoncu University in Gaziantep, Turkey, and evaluated.

All subjects were initially screened using the Fonseca anamnestic index (FAI), which has been validated in the Turkish population and assesses factors such as chewing, parafunctional habits, movement limitations, joint noise, and dizziness. ${ }^{15}$ A total of 389 individuals who were considered to have no dysfunction in the temporomandibular joint (i.e., had scores between 0 and 15), no primary and secondary headache, no toothache because of dental disease, and no denture problems, including pain or ill-fitting dentures, were included in the study. Individuals who had history of trauma to the face or the temporomandibular joint, history of whiplash, rheumatic disease, joint hypermobility, previous orthodontic or prosthodontic treatment, sleep-related conditions (e.g., obstructive sleep apnea syndrome), cognitive incapacity, medical disorders, or severe malocclusion or other malformations that may affect occlusion were excluded. They were divided into 3 groups in terms of age. Individuals aged 18-28 years (131 males, 104 females), 29-39 years ( 29 males, 56 females) and $40-50$ years ( 30 males, 39 females) were evaluated. The study was conducted between June 2018 and June 2019.

The physical characteristics and demographic information concerning the individuals were recorded before the test. The tone and viscoelastic properties of the masseter muscle were evaluated bilaterally in the supine 
position with the Myoton PRO device (Fig. 1). The mean value was calculated after taking 3 consecutive measurements at the measurement site for each parameter. The measurement site was assumed as the highest point of the muscle belly and was marked with a water-based pen to provide an anatomical reference point. The mandible was placed centrally with no contact between the upper and lower teeth. ${ }^{16}$

\section{Ethical approval}

Ethical approval was obtained from the ethics committee of the Hasan Kalyoncu University (decision No. 2019/61). The subjects were informed about the purpose and content of the study. Written consent was obtained from all individuals.

\section{Measurement tools}

\section{Myoton ${ }^{\circledR}$ PRO device}

The Myoton PRO device is a non-invasive, portable, hand-held myotonometer used to evaluate the viscoelastic properties of soft tissues. ${ }^{17}$ This device is known as cost-effective, reliable, valid, and easy-to-use in evaluating the mechanical properties of the musculoskeletal system. ${ }^{18,19}$ The muscle tone and viscoelastic parameters (stiffness and elasticity) were assessed by taking myotonometric measurements bilaterally from the skin overlying the masseter by means of Myoton PRO. The examiner marked the masseter with a small ink dot.

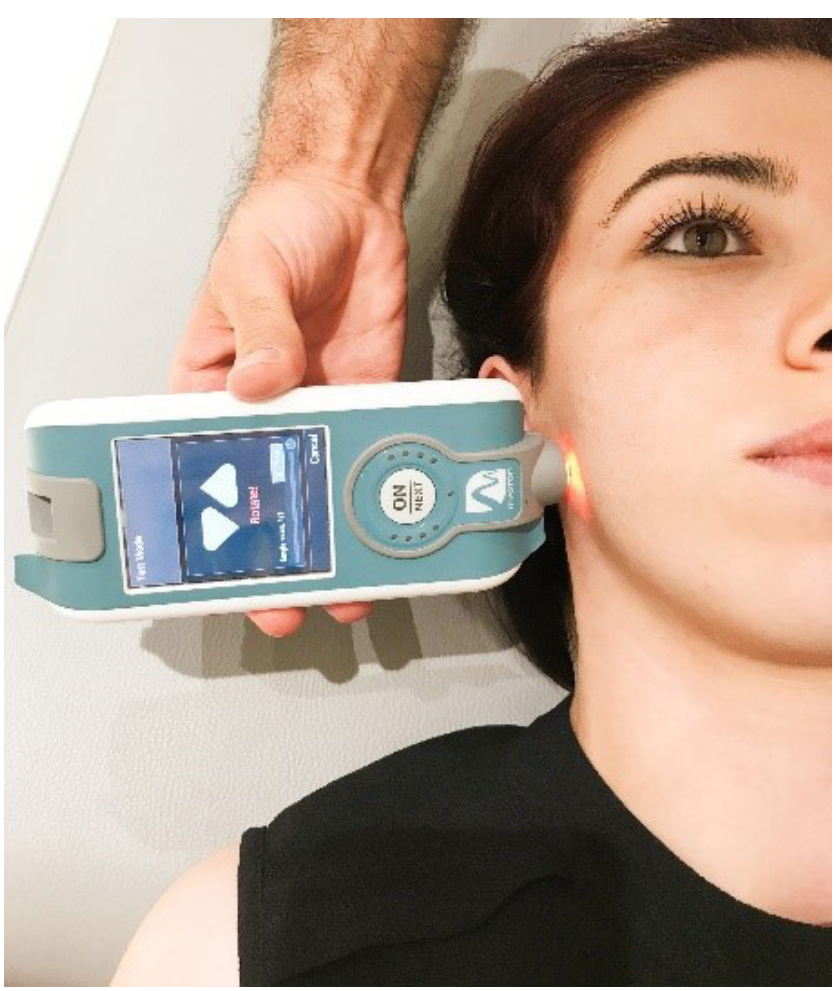

Fig. 1. Evaluation of the mechanical properties of the masseter muscle
This device has shown good-to-excellent reliability in healthy individuals,${ }^{20}$ the elderly, ${ }^{12}$ cancer $^{21}$ and stroke patients, ${ }^{22}$ athletes, ${ }^{23}$ and patients with neurodegenerative disease. ${ }^{24}$ The device measures the mechanical oscillations of the assessed soft tissues, induced by a mechanical impulse that is of a short duration $(15 \mathrm{~ms})$ and involves constant mechanical force (up to $0.6 \mathrm{~N}$ ). Measuring the mechanical oscillations occurring due to the mechanical impulse yields the following data: oscillation frequency [Hz]; logarithmic decrement (elasticity); and stiffness $[\mathrm{N} / \mathrm{m}] .{ }^{25}$ Resting muscle tone is the elastic and/or viscoelastic stiffness in the absence of contractile activity. Elasticity is the property whereby a body, when deformed by the applied load, recovers its previous configuration when the load is removed. The applied force is proportional to the strain within the elastic limit. Stiffness is a biomechanical feature of a muscle that characterizes its resistance to contraction or an external force that disrupts its initial state. ${ }^{26}$

\section{Fonseca anamnestic index}

Fonseca et al. developed the Fonseca anamnestic index (FAI), which is a 10-item instrument that allows the assessment of jaw function limitation, pain frequency, psychological distress, and the parafunctional behaviors related to TMD. ${ }^{27}$ The cross-cultural adaptation of the English version of FAI was completed in 2020 by Kaynak et al. ${ }^{15}$ The specificity was $83.7 \%$ and the sensitivity $-93.57 \%$. They found that FAI had a high level of accuracy (AUC (area under curve) $=0.928 ; 95 \% C I$ (confidence interval $)=0.890-20.964)$, a high sensitivity value (131 individuals with TMD diagnosed by means of FAI, compared to 140 individuals with TMD diagnosed by the dentist), and a high specificity value (54 individuals with no TMD diagnosed by means of FAI, compared to 65 individuals with no TMD diagnosedby the dentist and the physical therapist). The results demonstrated that the Turkish version of FAI (FAI-T) had good-to-excellent test-retest reliability and a high level of internal consistency, and provided considerable evidence that FAI-T could be used as a screening tool for the identification of TMD. ${ }^{15}$ Stasiak et al. stated that FAI was very sensitive (97.21\%) in identifying patients who actually had TMD, but not very specific (26\%) in identifying non-TMD patients, thus being indicated for use in the initial screening of patients only. ${ }^{28}$

\section{Data analyses}

The power analysis was performed with the use of the $G^{*}$ Power software, v. 3.1.9.2 (www.psycho. uni-duesseldorf.de/abteilungen/aap/gpower3), based on the expectation of a large effect size $(f=0.40)$ for comparisons among 3 age groups in terms of numerical variables for each gender $(\alpha=0.05 ; 1-\beta=0.80)$. 
The minimum required total sample size was estimated as 66 for 3 groups and 22 for each age group. The IBM SPSS Statistics for Windows software, v. 24.0 (IBM Corp., Armonk, USA), was used to analyze the data. The normal distribution of the data was determined with the Shapiro-Wilk test. The Mann-Whitney $U$ test was used to make comparisons between 2 groups. Since the number of groups was more than 2, the Kruskal-Wallis test was used to compare non-normally distributed variables. If, according to the Kruskal-Wallis test, the $p$-value was found to be significant, multiple comparison tests were used to determine the source of the difference. Also, Spearman's rank correlation test was used to determine the relationships between numerical variables. A $p$-value of less than 0.05 was considered statistically significant.

\section{Results}

The mean age of all individuals was $28.64 \pm 9.68$ years. The demographic characteristics of the participants are given in Table 1.

It was found that there were differences between genders in terms of viscoelastic measurements and tone of the right and left masseter muscles. The tone of the right and left masseter muscles was higher in men than in women ( $p=0.185$ and $p=0.035$, respectively). The measurements on both sides were similar in both genders in terms of stiffness $(p>0.05)$. The elasticity of the muscle, in terms of logarithmic decrement, on the right and left sides was higher in females $(p<0.05)$ (Table 2$)$.

\section{Mechanical properties of the masseter muscle in all individuals}

According to the results of Dunn's multiple comparison test, the right and left masseter muscle tone and stiffness were significantly lower in the 18-28 years age group (group 1) as compared to the other 2 groups $(p<0.05)$ except for the right masseter muscle tone, where the difference was insignificant ( $p=0.051)$, and there was no significant difference between the age groups of 29-39 years (group 2) and 40-50 years (group 3) $(p>0.05)$. The right and left masseter muscle elasticity was significantly higher in group 1 than in the other 2 groups $(p<0.05)$, for the right masseter muscle, it was similar between groups 2 and 3 $(p>0.05)$, whereas for the left masseter muscle, it was significantly higher in group 2 than in group $3(p<0.05)$. In light of these results, it can be said that as age increases, the masseter muscle tone and stiffness increase, and the elasticity of the muscle (logarithmic decrement - if the value increases, elasticity decreases) decreases (Table 3 and Table 4).

\section{Mechanical properties of the masseter muscle in male individuals}

Based on Dunn's multiple comparison test results for males, it was observed that the group 1 values for the right and left masseter muscle tone were significantly lower than in group 2 and group $3(p<0.05)$. For the right masseter muscle, the tone values were significantly higher in group 2 than in group $3(p<0.05)$, whereas

Table 1. Demographic characteristics of the participants

\begin{tabular}{|l|c|c|c|c|c|}
\multicolumn{1}{|c|}{ Characteristic } & $\begin{array}{c}\text { All individuals } \\
(N=389)\end{array}$ & $\begin{array}{c}\text { Males } \\
(n=190)\end{array}$ & $\begin{array}{c}\text { Females } \\
(n=199)\end{array}$ & Z & $p$-value \\
\hline Age $[$ years] & $28.64 \pm 9.68$ & $27.29 \pm 9.57$ & $29.92 \pm 9.63$ & -3.064 & $0.002^{*}$ \\
Height $[\mathrm{m}]$ & $1.69 \pm 0.86$ & $1.75 \pm 0.07$ & $1.64 \pm 0.06$ & -13.614 & $0.001^{*}$ \\
Weight $[\mathrm{kg}]$ & $71.91 \pm 14.42$ & $76.11 \pm 13.98$ & $67.90 \pm 13.72$ & -5.399 & $0.001^{*}$ \\
BMl $\left[\mathrm{kg} / \mathrm{m}^{2}\right]$ & $25.14 \pm 4.58$ & $24.87 \pm 4.06$ & $25.40 \pm 5.02$ & -0.827 & 0.408 \\
\hline
\end{tabular}

$\mathrm{BMI}$ - body mass index; ${ }^{*}$ statistically significant.

Data presented as mean \pm standard deviation $(M \pm S D)$.

Table 2. Tone, stiffness and elasticity of the right and left sides of the masseter muscle in all individuals, and distribution in terms of gender

\begin{tabular}{lc|c|c|c|c|}
\multicolumn{1}{c|}{ Variable } & $\begin{array}{c}\text { All individuals } \\
(N=389)\end{array}$ & $\begin{array}{c}\text { Males } \\
(n=190)\end{array}$ & $\begin{array}{c}\text { Females } \\
(n=199)\end{array}$ & Z -value \\
\hline MMTR [Hz] & $14.87 \pm 2.12$ & $15.10 \pm 2.40$ & $14.65 \pm 1.78$ & -1.325 & 0.185 \\
MMSR [N/m] & $283.78 \pm 70.79$ & $280.25 \pm 79.68$ & $287.16 \pm 61.11$ & -2.772 & 0.060 \\
MMDR [log] & $1.78 \pm 0.33$ & $1.73 \pm 0.33$ & $1.82 \pm 0.31$ & -3.277 & $0.001^{*}$ \\
MMTL [Hz] & $14.62 \pm 1.83$ & $14.82 \pm 1.87$ & $14.44 \pm 1.77$ & -2.107 & $0.035^{*}$ \\
MMSL [N/m] & $280.16 \pm 57.14$ & $277.33 \pm 59.86$ & $282.86 \pm 54.43$ & -1.527 & 0.127 \\
MMDL [log] & $1.76 \pm 0.34$ & $1.69 \pm 0.33$ & $1.82 \pm 0.34$ & -4.135 & $0.001^{*}$ \\
\hline
\end{tabular}

MMTR - right masseter muscle tone; MMSR - right masseter muscle stiffness; MMDR - right masseter muscle elasticity; MMTL - left masseter muscle tone; MMSL - left masseter muscle stiffness; MMDL - left masseter muscle elasticity; * statistically significant.

Data presented as $M \pm S D$. 
Table 3. Comparison of age groups in terms of tone, stiffness and elasticity of the right and left sides of the masseter muscle in all individuals and according to gender

\begin{tabular}{|c|c|c|c|c|c|c|}
\hline Gender & Variable & $\begin{array}{c}18-28 \text { years } \\
(n=235)\end{array}$ & $\begin{array}{c}29-39 \text { years } \\
(n=85)\end{array}$ & $\begin{array}{c}40-50 \text { years } \\
(n=69)\end{array}$ & $x^{2}$ & $p$-value \\
\hline \multirow{6}{*}{ All individuals } & MMTR [Hz] & $14.52 \pm 1.96$ & $15.21 \pm 2.13$ & $15.65 \pm 2.38$ & 22.25 & $0.001^{*}$ \\
\hline & MMSR [N/m] & $267.82 \pm 66.93$ & $308.40 \pm 61.67$ & $307.80 \pm 79.08$ & 66.52 & $0.001^{*}$ \\
\hline & MMDR [log] & $1.64 \pm 0.28$ & $1.90 \pm 0.21$ & $2.08 \pm 0.33$ & 115.80 & $0.001^{*}$ \\
\hline & MMTL $[\mathrm{Hz}]$ & $14.29 \pm 1.67$ & $15.05 \pm 1.88$ & $15.22 \pm 2.04$ & 22.44 & $0.001^{*}$ \\
\hline & MMSL [N/m] & $264.83 \pm 57.91$ & $298.32 \pm 48.98$ & $310.01 \pm 44.69$ & 72.11 & $0.001^{*}$ \\
\hline & MMDL [log] & $1.61 \pm 0.27$ & $1.86 \pm 0.27$ & $2.13 \pm 0.29$ & 128.81 & $0.001^{*}$ \\
\hline \multirow{6}{*}{ Males } & MMTR [Hz] & $14.75 \pm 2.01$ & $16.05 \pm 2.51$ & $15.72 \pm 2.79$ & 10.815 & $0.004^{*}$ \\
\hline & MMSR [N/m] & $265.24 \pm 70.50$ & $322.10 \pm 81.72$ & $305.33 \pm 96.08$ & 32.674 & $0.001^{*}$ \\
\hline & MMDR [log] & $1.62 \pm 0.28$ & $1.86 \pm 0.21$ & $2.07 \pm 0.38$ & 48.541 & $0.001^{*}$ \\
\hline & MMTL $[\mathrm{Hz}]$ & $14.48 \pm 1.70$ & $15.86 \pm 1.99$ & $15.29 \pm 2.08$ & 15.544 & $0.001^{*}$ \\
\hline & MMSL [N/m] & $262.99 \pm 56.39$ & $303.69 \pm 54.25$ & $314.47 \pm 56.65$ & 33.513 & $0.001^{*}$ \\
\hline & MMDL [log] & $1.58 \pm 0.26$ & $1.74 \pm 0.25$ & $2.12 \pm 0.32$ & 52.994 & $0.001^{*}$ \\
\hline \multirow{6}{*}{ Females } & MMTR [Hz] & $14.23 \pm 1.54$ & $14.77 \pm 1.77$ & $15.60 \pm 2.04$ & 18.997 & $0.001^{*}$ \\
\hline & MMSR [N/m] & $271.09 \pm 62.33$ & $301.30 \pm 47.53$ & $309.69 \pm 64.33$ & 31.892 & $0.001^{*}$ \\
\hline & MMDR [log] & $1.66 \pm 0.27$ & $1.92 \pm 0.21$ & $2.09 \pm 0.29$ & 58.975 & $0.001^{*}$ \\
\hline & MMTL $[\mathrm{Hz}]$ & $14.05 \pm 1.60$ & $14.63 \pm 1.69$ & $15.18 \pm 2.04$ & 15.011 & $0.001^{*}$ \\
\hline & MMSL [N/m] & $267.13 \pm 59.97$ & $295.54 \pm 46.28$ & $306.59 \pm 33.13$ & 36.891 & $0.001^{*}$ \\
\hline & MMDL [log] & $1.65 \pm 0.29$ & $1.92 \pm 0.25$ & $2.13 \pm 0.27$ & 68.451 & $0.001^{*}$ \\
\hline
\end{tabular}

* statistically significant (Kruskal-Wallis test).

Data presented as $M \pm S D$.

there was no significant difference between groups 2 and 3 for the left masseter muscle in terms of tone $(p>0.05)$. The right and left masseter muscle stiffness values in group 1 were significantly lower than in the other 2 groups $(p<0.05)$, and there was no significant difference between group 2 and group $3(p>0.05)$. The right and left masseter muscle elasticity in group 1 was significantly higher than in the other 2 groups $(p<0.05)$. Also, the right and left masseter muscle elasticity was significantly lower in group 3 than in group $2(p<0.05)$ (Table 3 and Table 4).

\section{Mechanical properties of the masseter muscle in female individuals}

Based on Dunn's multiple comparison test results for females, the right and left masseter muscle tone was significantly lower in group 1 than in the other 2 groups $(p<0.05)$, and both values were significantly lower in group 2 than in group $3(p<0.05)$. The right and left masseter stiffness values in group 1 were significantly lower than in the other 2 groups $(p<0.05)$, and there was no significant difference between group 2 and group 3 $(p>0.05)$. The right and left masseter muscle elasticity in group 1 was significantly higher than in the other 2 groups $(p<0.05)$, and the values in group 2 did not differ significantly from those in group $3(p>0.05)$ (Table 3 and Table 4).
Table 4. Dunn's multiple comparison test results for all individuals and both genders according to age group

\begin{tabular}{|c|c|c|c|c|}
\hline Gender & Variable & $\begin{array}{c}18-28 \text { years } \\
\text { vs } \\
29-39 \text { years } \\
p \text {-value }\end{array}$ & $\begin{array}{c}18-28 \text { years } \\
\text { vs } \\
40-50 \text { years } \\
p \text {-value }\end{array}$ & $\begin{array}{c}29-39 \text { years } \\
\text { vs } \\
40-50 \text { years } \\
p \text {-value }\end{array}$ \\
\hline \multirow{6}{*}{$\begin{array}{l}\text { All } \\
\text { individuals }\end{array}$} & MMTR $[\mathrm{Hz}]$ & $0.003^{*}$ & 0.051 & 0.414 \\
\hline & MMSR [N/m] & $0.001^{*}$ & $0.001^{*}$ & 0.565 \\
\hline & MMDR [log] & $0.001^{*}$ & $0.001^{*}$ & 0.189 \\
\hline & MMTL $[\mathrm{Hz}]$ & $0.001^{*}$ & $0.026^{*}$ & 0.265 \\
\hline & MMSL [N/m] & $0.001^{*}$ & $0.001^{*}$ & 0.438 \\
\hline & MMDL [log] & $0.005^{*}$ & $0.001^{*}$ & $0.001^{*}$ \\
\hline \multirow{6}{*}{ Males } & MMTR [Hz] & $0.042^{*}$ & $0.001^{*}$ & $0.024^{*}$ \\
\hline & MMSR [N/m] & $0.001^{*}$ & $0.001^{*}$ & 0.129 \\
\hline & MMDR [log] & $0.001^{*}$ & $0.001^{*}$ & $0.027^{*}$ \\
\hline & MMTL $[\mathrm{Hz}]$ & $0.023^{*}$ & $0.001^{*}$ & 0.129 \\
\hline & MMSL [N/m] & $0.001^{*}$ & $0.001^{*}$ & 0.079 \\
\hline & MMDL [log] & $0.001^{*}$ & $0.001^{*}$ & $0.005^{*}$ \\
\hline \multirow{6}{*}{ Females } & MMTR $[\mathrm{Hz}]$ & $0.001^{*}$ & $0.001^{*}$ & $0.001^{*}$ \\
\hline & MMSR [N/m] & $0.003^{*}$ & $0.001^{*}$ & 0.196 \\
\hline & MMDR [log] & $0.001^{*}$ & $0.001^{*}$ & 0.473 \\
\hline & MMTL $[\mathrm{Hz}]$ & $0.001^{*}$ & $0.001^{*}$ & $0.013^{*}$ \\
\hline & MMSL [N/m] & $0.001^{*}$ & $0.001^{*}$ & 0.485 \\
\hline & MMDL [log] & $0.001^{*}$ & $0.001^{*}$ & 0.066 \\
\hline
\end{tabular}

\footnotetext{
* statistically significant.
} 
A weak positive correlation was found between the right and left masseter muscle tone and age $(r=0.307$ and $r=0.325$, respectively; $p=0.001$ ). There was a moderate positive correlation between the right and left masseter muscle stiffness and age $(r=0.507$ and $r=0.511$, respectively; $p=0.001)$. A strong positive correlation was observed between the right and left masseter muscle elasticity and age $(r=0.614$ and $r=0.645$, respectively; $p=0.001)$.

\section{Discussion}

The major findings of this study, in which we examined the tone and viscoelastic parameters of the masseter muscle in healthy individuals as well as their relationship with age and gender, and aimed to specify the reference values for the muscle, are that the tone of the masseter muscle is higher in males, its elasticity (logarithmic decrement) is higher in females, the stiffness of the muscle is similar in both genders, and the elasticity of the muscle decreases with aging.

It is unavoidable that some changes occur in the muscle structure due to aging. One of the changes is the reduction of the contractile areas of the muscle due to the transformation of contractible fibers into non-contractile areas. However, muscle stiffness tends to increase with aging. Changes in the distribution of muscle fibers are also observed with aging. These changes are characterized by the loss of type II fibers and an increase in type I fibers. As a result, an increase in muscle stiffness occurs, as type I fibers cause more stiffness than type II fibers. These age-related changes have been investigated in several studies. ${ }^{29,30}$ Following the previous findings in the literature, we also found that the tone and stiffness of the muscle increase, and its elasticity decreases with aging.

It is known that the total muscle mass in women is 15-20\% lower than in men. At the same time, women have weaker muscle tone and strength. ${ }^{31,32}$ In terms of jaw muscles, it has been reported that the jaw muscles in men are stronger than in women, and there is a positive correlation between testosterone levels and mouth closing force. $^{33}$ In an experimental mouse model study, it was shown that the masseter muscle mass increases by nearly $38 \%$ with the supplementation of testosterone. ${ }^{34}$ It has been noted in studies that testosterone has a significant effect on the masseter muscle mass. ${ }^{34}$ In our study, the tone of the masseter muscle was found to be higher in males than in females, and we think that this result may be due to the effects of sex hormones.

Muscle tendons are weaker and looser in women; this situation causes more joint mobility in women. In other words, elasticity and joint mobility are higher in women. ${ }^{32}$ We think that the higher elasticity (logarithmic decrement) of the masseter muscle in the female group in our study can be explained by the differences in the musculoskeletal system of males and females.

Myotonometric measurement results may vary, depending on the position (horizontal or vertical) of the muscles. ${ }^{24}$ However, in a study evaluating the stiffness of the masseter muscle in the sitting position, no difference was found between genders. ${ }^{35}$ In our study, the measurements were made in the supine position, where there is no activity against gravity. In accordance with the literature, we found that the stiffness of the masseter muscle was similar in both genders.

There are studies in the literature that observed a difference or no difference between the tone and viscoelastic values of the right and left masseter muscle. ${ }^{36,37}$ In our study, differences were observed between the values of some parameters of the right and left masseter muscles. We think that these differences could be explained by dominance.

\section{Limitations}

The fact that the temporal muscle was not included in the measurements of our study could be viewed as a limitation. Also, all participants came from the same university, and this selection method from the same participant pool was a limitation of our study with regard to sample selection. Although FAI is a simple, quick and easy way to determine myogenic TMD, it is not the gold standard for the clinical evaluation. Bruxism and TMD cases were excluded from the study; they were determined through the FAI questions based upon people's statements. Individuals diagnosed with sleep problems, such as obstructive sleep apnea, were not included in the study. However, since such problems are difficult to diagnose even by a physician due to their complex etiology, their presence was determined based upon people's statements. These are the limitations of our study. In the future, multidisciplinary studies can be designed based on the samples diagnosed by dentists.

\section{Conclusions}

The tone and viscoelastic parameters of the masseter muscle can be examined using different evaluation methods to compare pre- and post-treatment changes in the muscle in fields such as plastic surgery, dentistry, physical therapy and rehabilitation, and speech and language therapy. We hope that the results of our study can provide the reference values for studies in these fields.

\section{ORCID iDs}

Engin Ramazanoglu (D) https://orcid.org/0000-0002-2765-0121 Begumhan Turhan (D) https://orcid.org/0000-0003-0842-2059 Serkan Usgu (iD https://orcid.org/0000-0002-4820-9490 


\section{References}

1. Iguchi H, Magara J, Nakamura $\mathrm{Y}$, Tsujimura T, Ito K, Inoue M. Changes in jaw muscle activity and the physical properties of foods with different textures during chewing behaviors. Physiol Behav. 2015;152(Pt A):217-224. doi:10.1016/j.physbeh.2015.10.004

2. Lin CS, Wu CY, Wu SY, et al. Age- and sex-related differences in masseter size and its role in oral functions. J Am Dent Assoc. 2017;148(9):644-653. doi:10.1016/j.adaj.2017.03.001

3. Mapelli A, Zanandréa Machado BC, Giglio LD, Sforza C, De Felício CM Reorganization of muscle activity in patients with chronic temporomandibular disorders. Arch Oral Biol. 2016;72:164-171. doi:10.1016/j.archoralbio.2016.08.022

4. Ariji Y, Ariji E. Magnetic resonance and sonographic imagings of masticatory muscle myalgia in temporomandibular disorder patients. Jpn Dent Sci Rev. 2017;53(1):11-17. doi:10.1016/j.jdsr.2016.05.001

5. Cho GH, Lee $\mathrm{Y}$. Analysis of masticatory muscle activity based on presence of temporomandibular joint disorders. Med Sci Monit. 2020;26:e921337. doi:10.12659/MSM.921337

6. Manzotti A, Viganoni C, Lauritano D, et al. Evaluation of the stomatognathic system before and after osteopathic manipulative treatment in 120 healthy people by using surface electromyography. Int J Environ Res Public Health. 2020;17(9):3250. doi:10.3390/ijerph17093250

7. Hara K, Namiki C, Yamaguchi K, et al. Association between myotonometric measurement of masseter muscle stiffness and maximum bite force in healthy elders. J Oral Rehabil. 2020;47(6):750-756. doi:10.1111/joor.12968

8. Ohara $\mathrm{Y}$, Hirano $\mathrm{H}$, Watanabe $\mathrm{Y}$, et al. Masseter muscle tension and chewing ability in older persons. Geriatr Gerontol Int. 2013;13(2):372-377. doi:10.1111/j.1447-0594.2012.00909.x

9. Correa-de-Araujo R, Harris-Love MO, Miljkovic I, Fragala MS, Anthony BW, Manini TM. The need for standardized assessment of muscle quality in skeletal muscle function deficit and other aging-related muscle dysfunctions: A symposium report. Front Physiol. 2017;8:87. doi:10.3389/fphys.2017.00087

10. Barros BM, Biasotto-Gonzalez DA, Bussadori SK, Fidelis de Paula Gomes CA, Politti F. Is there a difference in the electromyographic activity of the masticatory muscles between individuals with temporomandibular disorder and healthy controls? A systematic review with meta-analysis. J Oral Rehabil. 2020;47(5):672-682. doi:10.1111/joor.12941

11. Masuda $M$, lida $T$, Exposto $F G$, et al. Referred pain and sensations evoked by standardized palpation of the masseter muscle in healthy participants. J Oral Facial Pain Headache. 2018;32(2):159-166. doi:10.11607/ofph.2019

12. Aird L, Samuel D, Stokes M. Quadriceps muscle tone, elasticity and stiffness in older males: Reliability and symmetry using the MyotonPRO. Arch Gerontol Geriatr. 2012;55(2):e31-e39. doi:10.1016/j. archger.2012.03.005

13. Leonard CT, Deshner WP, Romo JW, Suoja ES, Fehrer SC, MikhailenokEL. Myotonometer intra- and interrater reliabilities. Arch Phys Med Rehabil. 2003;84(6):928-932. doi:10.1016/s0003-9993(03)00006-6

14. Leonard CT, Brown JS, Price TR, Queen SA, Mikhailenok EL. Comparison of surface electromyography and myotonometric measurements during voluntary isometric contractions. J Electromyogr Kinesiol. 2004;14(6):709-714. doi:10.1016/j.jelekin.2004.06.001

15. Kaynak BA, Tass S, Salkın Y. The accuracy and reliability of the Turkish version of the Fonseca anamnestic index in temporomandibular disorders. Cranio. 2020;1-6. doi:10.1080/08869634.2020.1812808

16. Wozniak E, Loster JE, Wieczorek A. Relation between headache and mastication muscle tone in adolescents. Pain Res Manag. 2018;ID 381973. doi:10.1155/2018/7381973

17. Gilbert I, Gaudreault N, Gaboury I. Intra- and inter-evaluator reliability of the MyotonPRO for the assessment of the viscoelastic properties of caesarean section scar and unscarred skin. Skin Res Technol. 2020. doi:10.1111/srt.12956

18. Feng YN, Li YP, Liu CL, Zhang ZJ. Assessing the elastic properties of skeletal muscle and tendon using shearwave ultrasound elastography and MyotonPRO. Sci Rep. 2018;8(1):17064. doi:10.1038/s41598-018-34719-7

19. Morgan G, Martin R, Welch H, Williams L, Morris K. Objective assessment of stiffness in the gastrocnemius muscle in patients with symptomatic Achilles tendons. BMJ Open Sport Exerc Med. 2019;5(1):e000622. doi:10.1136/bmjsem-2019-000622
20. Bailey L, Samuel D, Warner MB, Stokes M. Parameters representing muscle tone, elasticity and stiffness of biceps brachii in healthy older males: Symmetry and within-session reliability using the MyotonPRO. J Neurol Disord. 2013;1(1):1-7. doi:10.4172/jnd.1000116

21. Yeo SM, Kang H, An S, Cheong I, Kim Y, Hwang JH. Mechanical properties of muscles around the shoulder in breast cancer patients: Intra-rater and inter-rater reliability of the MyotonPRO. PM $R$. 2020;12(4):374-381. doi:10.1002/pmrj.12227

22. Lo WLA, Zhao JL, Li L, Mao YR, Huang DF. Relative and absolute interrater reliabilities of a hand-held myotonometer to quantify mechanical muscle properties in patients with acute stroke in an inpatient ward. Biomed Res Int. 2017;2017:4294028. doi:10.1155/2017/4294028

23. Schneebeli A, Falla D, Clijsen R, Barbero M. Myotonometry for the evaluation of Achilles tendon mechanical properties: A reliability and construct validity study. BMJ Open Sport Exerc Med. 2020;6(1):e000726. doi:10.1136/bmjsem-2019-000726

24. Zinder SM, Padua DA. Reliability, validity, and precision of a handheld myometer for assessing in vivo muscle stiffness. J Sport Rehabil. 2011;20(3):2010_0051. doi:10.1123/jsr.2010-0051

25. Taş S, Aktaş D. Menstrual cycle does not affect the mechanical properties of muscle and tendon. Muscles Ligaments Tendons $J$. 2020;10(1):11-16. doi:10.32098/mltj.01.2020.02

26. Simons GD, Mense $S$. Understanding and measurement of muscle tone as related to clinical muscle pain. J Pain. 1998;75(1):1-17. doi:10.1016/S0304-3959(97)00102-4

27. da Fonseca DM, Bonfante G, do Valle AL, Torres de Freitas SF. Diagnosis of the craniomandibular dysfunction through anamnesis [in Portuguese]. Rev Gauch de Odontol. 1994;4(1):23-32.

28. Stasiak G, Maracci LM, de Oliveira Chami V, et al. TMD diagnosis: Sensitivity and specificity of the Fonseca Anamnestic Index. Cranio. 2020;1-5. doi:10.1080/08869634.2020.1839724

29. Kocur P, Grzeskowiak M, Wiernicka M, Goliwas M, Lewandowski J, Łochyński D. Effects of aging on mechanical properties of sternocleidomastoid and trapezius muscles during transition from lying to sitting position - a cross-sectional study. Arch Gerontol Geriatr. 2017;70:14-18. doi:10.1016/j.archger.2016.12.005

30. Narici MV, Maffulli N. Sarcopenia: Characteristics, mechanisms and functional significance. Br Med Bull. 2010;95:139-159. doi:10.1093/ $\mathrm{bmb} / \mathrm{ldq008}$

31. Blair SN, LaMonte MJ, Nichaman MZ. The evolution of physical activity recommendations: How much is enough? Am J Clin Nutr. 2004;79(5):913S-920S. doi:10.1093/ajcn/79.5.913S

32. Ramazanoğlu E, Usgu S, Yakut Y. Assessment of the mechanical characteristics of the lower extremity muscles with myotonometric measurements in healthy individuals. Physiother Quart. 2020;28(4):1-12. doi:10.5114/pq.2020.97458

33. Mohr BA, Bhasin S, Kupelian V, Araujo AB, O'Donnell AB, McKinlay JB. Testosterone, sex hormone-binding globulin, and frailty in older men. JAm Geriatr Soc. 2007;55(4):548-555. doi:10.1111/j.1532-5415.2007.01121.x

34. Widmer CG, Morris-Wiman J. Limb, respiratory, and masticatory muscle compartmentalization: Developmental and hormonal considerations. Prog Brain Res. 2010;187:63-80. doi:10.1016/B978-0444-53613-6.00005-8

35. Dietsch AM, Clark HM, Steiner JN, Solomon NP. Effects of age, sex, and body position on orofacial muscle tone in healthy adults. J Speech Lang Hear Res. 2015;58(4):1145-1150. doi:10.1044/2015_JSLHR-S-14-0325

36. Olchowy A, Wieckiewicz M, Winocur E, et al. Great potential of ultrasound elastography for the assessment of the masseter muscle in patients with temporomandibular disorders. A systematic review. Dentomaxillofac Radiol. 2020;49(8):20200024. doi:10.1259/dmfr.20200024:2-9

37. Sukanya SA, Kamalanand K, Thayumanavan B, Emmanuel C, Rajnikanth V. Time domain analysis on myoelectric activity of masseter muscles in resting and chewing conditions. Netw Model Anal Health Inform Bioinform. 2020;9:18. doi:10.1007/s13721-020-0224-2 